\title{
FIREWORKS ON WEATHER RADAR AND CAMERA
}

Adapted From "Fireworks On Weather Radar and Camera," by Dušan S. Zrnić (National Severe Storms Laboratory), Pengfei Zhang, Valery Melnikov, and Eric Kabela. Published online in BAMS, February 2020. For the full, citable article, see DOI:10.1175/BAMS-D-18-0248.1.

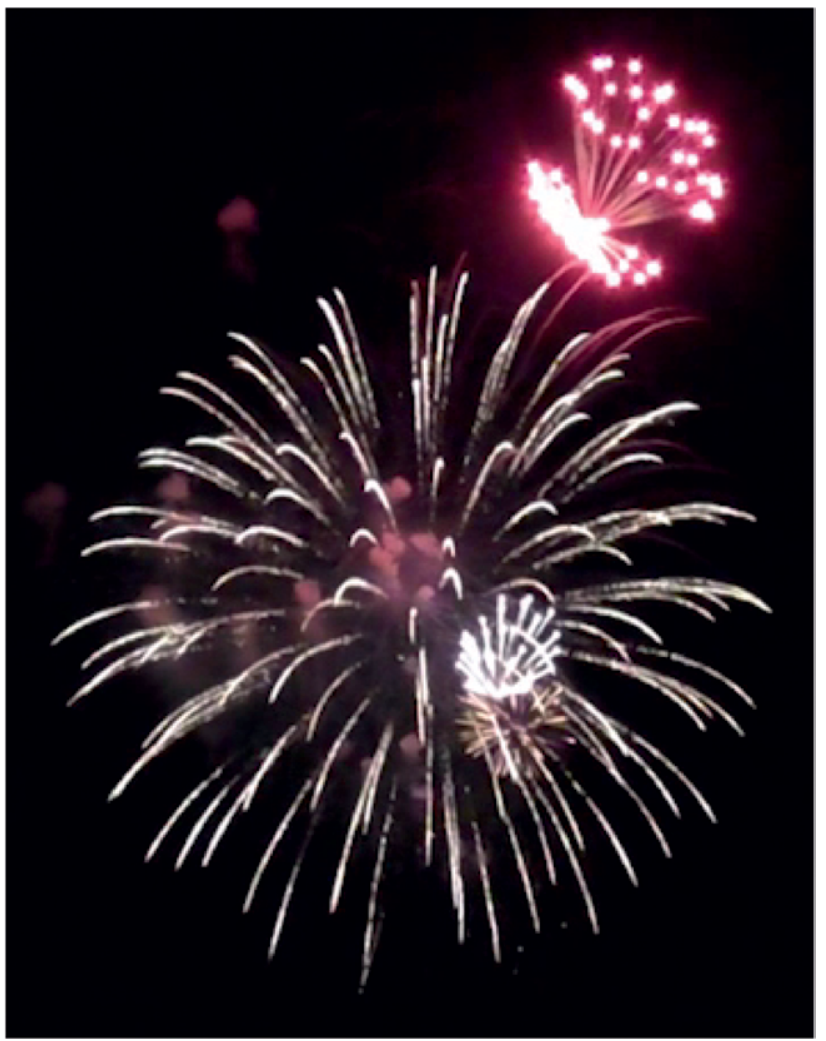

- he upgrade of the WSR-88D network to dual polarization adds new capabilities to operational weather radars-namely, identification of different scatterer types, further improvement in quantitative precipitation estimation, and potent depiction of storms. In addition, applications have broadened beyond those initially intended. Radars have become indispensable tools in the arsenal of aeroecologists: dual polarization makes possible discrimination between insects and birds. The radars can help identification of volcanic ash and tornadic debris, and detection and tracking of smoke plumes from forest fires. With dual polarization, the average delay in detection of fire onset is $5 \mathrm{~min}$, compared to the 15-min delay considered the low limit for good conventional systems.

An upgrade to multifunction phased array radar would serve weather and aviation needs, but also enhance homeland security by detecting noncooperative 
flying threats and ground-based explosions. Locating the explosive release of harmful chemicals may be sufficient to provide-via numerical models-downwind conditions and warnings to the public of impending hazards, even when the plume reflectivity is too weak for tracking.

Observations of Norman, Oklahoma, municipal fireworks on 4 July 2017 from multiple weather surveillance radars and high-speed photography provide a reasonable test of this capability to detect and track explosions and plumes. The fireworks were captured from close range with the National Severe Storms Laboratory (NSSL) X-band experimental dual-polarization 3-cm wavelength radar (XERES) and high-speed camera. Additional data from a nearby Terminal Doppler Weather Radar (TDWR) (5-cm wavelength) and WSR-88D (10-cm wavelength) are used for comparison of firework characteristics. The Norman fireworks have smaller caliber shells than fireworks in major cities. Therefore, fireworks from Fort Worth, Texas, observed by a nearby WSR-88D are presented for additional comparison.

We placed XERES close to the launch site and a high-resolution, high-speed video camera next to it. The images capture the height, evolution, and duration of individual fireworks bursts. During the 15-minute show, XERES completed approximately 15 volume scans that took $1 \mathrm{~min}$ each.

\section{Visual observations}

For an average peony firework (a flower-like explosion that quickly turns into a bright, explosive bouquet) immediately after explosion, visible burning segments called "stars" are on a spherical surface expanding uniformly. From our images, individual stars become identifiable between 0.02 and $0.04 \mathrm{~s}$ after the burst onset; the firework remains visible for approximately $1 \mathrm{~s}$. The largest diameter of this burst is about $100 \mathrm{~m}$ and the number of stars we counted is 121. The height of explosion is $190 \mathrm{~m}$ above ground. Many similar fireworks were part of the show. Stars in commercial fireworks range from about $12 \mathrm{~mm}$ to more than $18 \mathrm{~mm}$.

Video showed that the terminal velocities of stars in peony bursts (the most frequent) were about $14-16 \mathrm{~m} \mathrm{~s}^{-1}$. A very few stars stuck together fall at about $7 \mathrm{~m} \mathrm{~s}^{-1}$. We estimated high

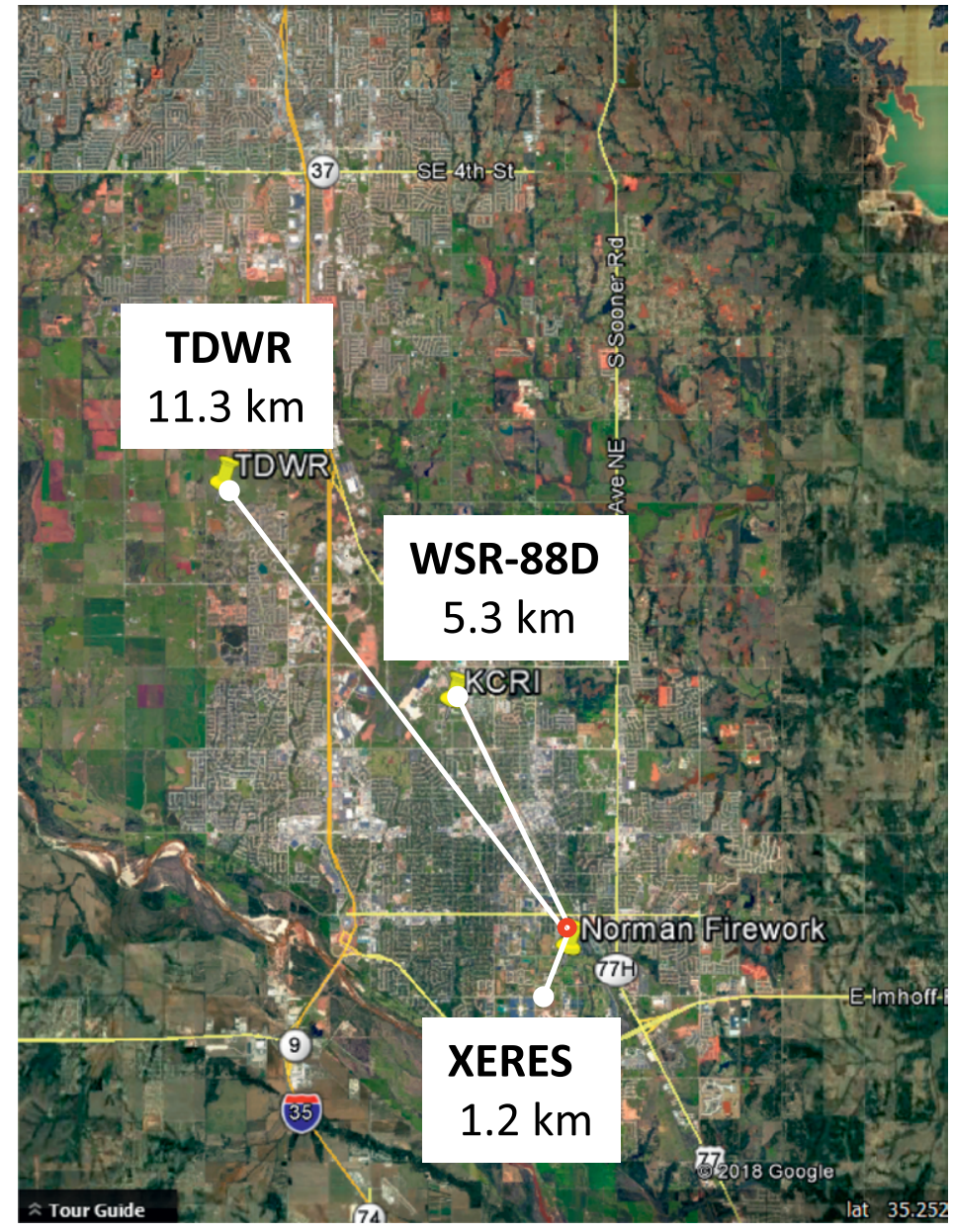

\section{$\Delta$}

Lations of the Terminal Doppler Weather Radar (5.35-cm wavelength) serving the Oklahoma City Airport, the WSR-88D (10-cm wavelength) in Norman supporting the network of National Weather Service radars, the XERES research mobile radar $(3.2-\mathrm{cm}$ wavelength), and the fireworks (red circle). The ranges from the radars to the fireworks location are indicated. The image is from Google Earth. terminal velocities of $22 \mathrm{~m} \mathrm{~s}^{-1}$ in a burst that looks like a weeping willow. By comparison, the 90th percentile terminal velocity is $10 \mathrm{~m}$ $\mathrm{s}^{-1}$ for $1-\mathrm{cm}$ hail and $20 \mathrm{~m} \mathrm{~s}^{-1}$ for $4-\mathrm{cm}$ hail. The stars of similar size likely experience less drag and fall faster. This suggests that star sizes on the order of $1 \mathrm{~cm}$ are a reasonable estimate for Norman fireworks.

Observed burst diameters are mostly between 100 and $150 \mathrm{~m}$, and few exceed $200 \mathrm{~m}$. Duration of the visible effects of small bursts is 1-2 s, and large bursts last 5-6 s. Many chrysanthemum-pattern fireworks were part of the show, and the recommended burning time for their stars is less than $5 \mathrm{~s}$. The average number of bursts per minute is about 33. Of these, $88 \%$ occurred between 100 and $200 \mathrm{~m}$ off the ground, $8 \%$ at heights above $200 \mathrm{~m}$, and $4 \%$ below $50 \mathrm{~m}$.

Visual observations indicate that stars replenish the volume above the launch site almost continuously. Fireworks are launched on average every $2 \mathrm{~s}$; nonetheless, fireworks 
are launched in bunches very frequently so some bursts overlap in space and time. Additionally, the rockets carrying the shell may crisscross the resolution volumes. The largest number of stars we counted is 325 in a peony. Most large values are 200-300. In cases of overlapping bursts, the number of stars in the radar resolution volume could be $2-3$ times more. The number and the location within the resolution volume determine the reflectivity. Beyond fireworks, the vertical (RHI) cross section of $Z_{\mathrm{DR}}$ exhibits a -2 to 4 $\mathrm{dB}$ range, implying the presence of diverse scatterers such as biota composed of insects and/or birds and bats. None of the polarimetric variables has information for unambiguously distinguishing the fireworks from the background biota.

\section{Observation with the other radars}

Scans at elevation angles of $0.5^{\circ}$ and $1.0^{\circ}$ by the TDWR yield information from 50 to $200 \mathrm{~m}$ above the ground. Volume scans take $6 \mathrm{~min}$; therefore, three are completed during the 15 -min firework show. There are six scans of $0.5^{\circ}$ data and three scans of $1.0^{\circ}$ data.

Reflectivity peaks above the location of fireworks in each of the six $0.5^{\circ}$ elevation scans and each of the three $1^{\circ}$ elevation scans. The increase and persistence of the signal distinguish it from the reflectivities in the environment.

The WSR-88D captured fireworks data in all its low-elevation scans: four scans at $0.5^{\circ}$ elevation separated by $3.5 \mathrm{~min}$, and two at $0.9^{\circ}$ separated by $6 \mathrm{~min}$. In contrast to the TDWR observation, the reflectivity peak of fireworks is indistinguishable from similar sporadic peaks of other scatterers. Only time persistence of enhanced reflectivity over the fireworks location and continuity in the lowest two elevations indicates that an anomaly is present.

\section{Comparisons}

In the XERES data at the highest elevation $\left(10^{\circ}\right.$ and beam center at $210 \mathrm{~m}), 10 \%$ fewer scans had evidence of fireworks (i.e., enhanced $Z$ ) than at the lower elevations. This could be because fewer bursts occurred at that height and these were seldom overlapping and hence were missed due to the 1-min volume scan revisit time. Average reflectivity decreases with height for all three radars.

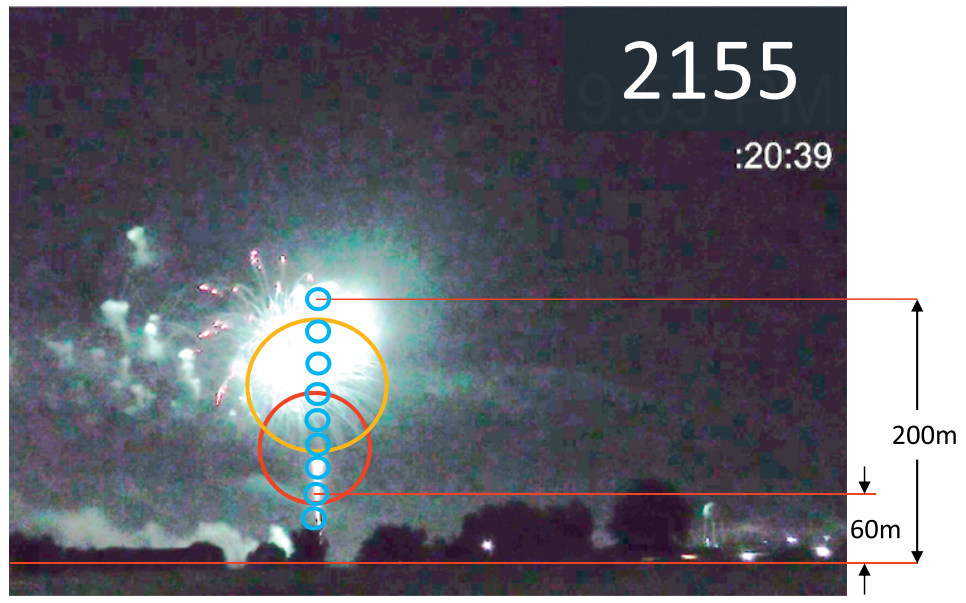

$\Delta$ * View of fireworks in Reeves Park, Norman, from the location of the XERES. The circles depict radar beam cross sections. The centers of the beam cross sections of the XERES radar are at the heights of its elevation scans and the cross sections (blue circles) are in the plane of the image. The cross sections of the other radar beams are for their lowest elevation $\left(0.5^{\circ}\right)$ scans and cut the image plane but are plotted in it for reference. The circle colors refer to radars as follows: XERES (blue), WSR-88D (red), TDWR (yellow). The indicated heights are above ground level (AGL) at the fireworks location. The bottom red line marks the ground level at the launch site, which happens to be the same as the ground level at the XERES location. The time at the top right is CDT hours and minutes. The time below it relates to the seconds and hundredths of seconds for this snapshot.

The spread of $Z$ and the maximum values measured by XERES are the largest of the three. XERES has at least 10 times more data points at each elevation, and its resolution volume is much smaller.

The high (33-39 dBZ) Zs from fireworks are much larger than the $20 \mathrm{dBZ}$ in smoke from wildfires and apartment fires. The scatterers in these plumes are millimeter-size oriented particles, which produce spread of $Z_{\mathrm{DR}}$ between -2 and $7.9 \mathrm{~dB}$, similar to the spread from fireworks.

\section{Fireworks in Fort Worth}

Fort Worth has the largest fireworks display in northern Texas. The 2018 show lasted about 30 minutes and the location of the launch was $20.5 \mathrm{~km}$ from the WSR-88D. 
Four consecutive peak $Z$ values were bigger than $40 \mathrm{~dB} Z$ at the $0.5^{\circ}$ elevation. The maximum, $47.5 \mathrm{dBZ}$, would correspond to about $34 \mathrm{~mm} \mathrm{~h}^{-1}$ of rain. The beam center over the launch site is $254 \mathrm{~m}$ above ground, slightly more than the 230-m altitude of the beam center in the highest of the Norman scans. Obviously, the Fort Worth fireworks are produced by significantly larger and more powerful shells. The reflectivities are, on average, larger by about $20 \mathrm{~dB}$ than the reflectivities of the Norman fireworks.

\section{Discussion}

Unlike smoke plumes generated by fires, the fireworks produce only a small amount of visible smoke. Moreover, we have no evidence of lightweight $\mathrm{mm}$ to $\mathrm{cm}$ size particles, which are common in ordinary fires. The stars, however, reflect sufficient energy, which weather radars can detect. By analogy, we expect similar detection of explosions. Pinpointing locations would be useful to first responders or air quality forecasters.

The National Weather Service WSR-88D
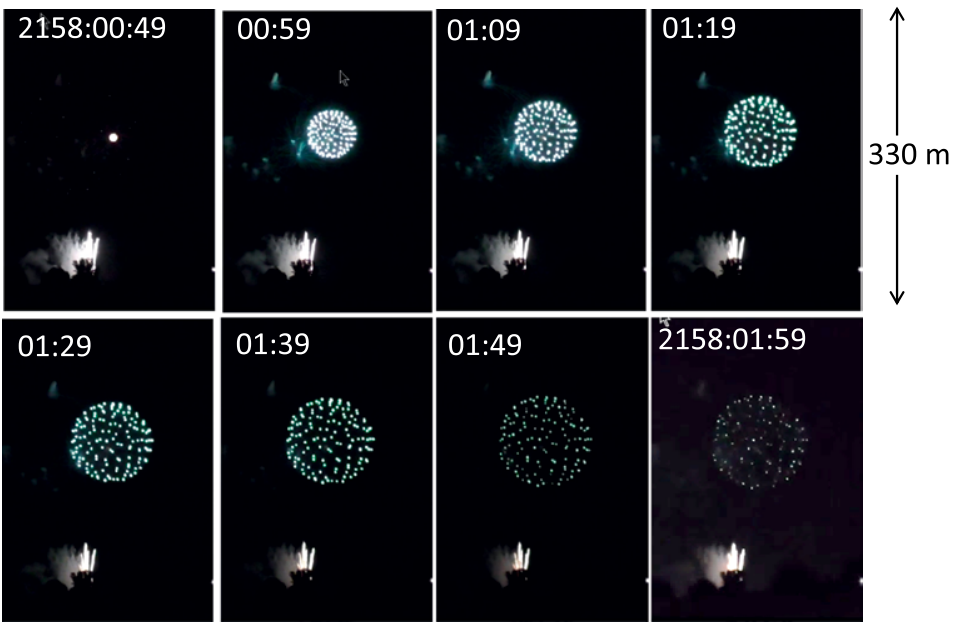

* Time sequence of a burst in 0.1-s intervals starting at 2158:00:49 CDT. The first and second digits in the last pair of the time stamp are tenth and hundredth of a second. data at multiple wavelengths can reveal the spatial extent of the fireworks and the heights of bursts. Moreover, it is possible to roughly infer the size of significant stars and components of their velocity. The radar display of polarimetric variables is itself a colorful spectacle. Using smartphones, the public can enjoy radar images and the real thing at the same time. $\therefore$

\section{METADATA}

BAMS: What would you like readers to learn from this article?

Dusan Zrnić: Operational, polarimetric radars detect fireworks. Also, by comparing reflectivity at three wavelengths, we can roughly estimate the dominant size of "stars" of fireworks.

BAMS: Was this a surprise?

Zrnic: We expected that the polarimetric variables would detect the bursts, but we were surprised by the high values of reflectivities: $47 \mathrm{dBZ}$ from large metropolitan displays versus $39 \mathrm{dBZ}$ for small municipal fireworks as in Norman. These high reflectivity values can bias rainfall measurements unless they are eliminated from further processing.

BAMS: Why study fireworks on radar?
DZ: Initially we were trying to identify onsets and locations of fires and explosions. We found we could do this using historic WSR-88D data, but not very well. Then my coauthor, Valery Melnikov, suggested that fireworks could be a proxy for these events, and this turned out to be true. The obvious advantage is that the exact place and time of fireworks detonation is known, making it is easy to locate a mobile radar in a favorable position to obtain key data.

\section{BAMS: What else surprised you?}

Zrnic: The highest fall speeds of about $22 \mathrm{~m} \mathrm{~s}^{-1}$ exceeded our expectations. We also did not realize how transient the returns are; a firework can be seen by eye for up to several seconds and after that it turns into ash, which is not detectable by radar.
BAMS: What was the biggest challenge you encountered?

DZ: We were hoping we might be able to observe the dispersion of Doppler velocities in the Doppler spectra, and collected such data. Unfortunately, we lost these data. Another first for us was to learn how to use software for displaying visual images; once we learned, it became a matter of time to do the analysis. Also, to develop the backscattering model of "stars" required extensive literature search. There is no information about the refractive index of "stars," so we had to look up the composition of these and estimate the values for mixtures of three ingredients. The good thing is that the results are not very sensitive to a range of possible values. 